\title{
Laser-driven formation of a high-pressure phase in amorphous silica
}

Alberto Salleo, ${ }^{1,2^{*}}$ Seth T. Taylor, ${ }^{2}$ Michael C. Martin, ${ }^{3}$ Wendy R. Panero, ${ }^{4,5}$ Raymond Jeanloz, ${ }^{4}$ François Y. Génin, ${ }^{6}$ and Timothy Sands. ${ }^{2}$

${ }^{1}$ Palo Alto Research Center, 3333 Coyote Hill Road, Palo Alto, CA 94304.

${ }^{2}$ Department of Materials Science and Engineering, University of California Berkeley, Berkeley, CA 94720.

${ }^{3}$ Advanced Light Source Division, Lawrence Berkeley National Laboratory, 1 Cyclotron Road, BLDG 6R2100, Berkeley, CA 94720-8226.

${ }^{4}$ Department of Earth and Planetary Science, University of California Berkeley, Berkeley, CA 94720.

${ }^{5}$ now at Department of Geological Sciences, University of Michigan, Ann Arbor, MI 48109.

${ }^{6}$ Lawrence Livermore National Laboratory, 7000 East Avenue, Livermore, CA94550.

* to whom correspondance should be addressed (asalleo@parc.com) 


\begin{abstract}
A combination of electron diffraction and infrared reflectance measurements shows that synthetic silica transforms partially into stishovite under high-intensity $\left(\mathrm{GW} / \mathrm{cm}^{2}\right)$ laser irradiation, probably by the formation of a dense ionized plasma above the silica surface. During the transformation the silicon coordination changes from four-fold to six-fold and the silicon-oxygen bond changes from mostly covalent to mostly ionic, such that optical properties of the transformed material differ significantly from those of the original glass. This phase transformation offers one suitable mechanism by which laser-induced damage grows catastrophically once initiated, thereby dramatically shortening the service lifetime of optics used for high-power photonics applications such as inertial confinement fusion.
\end{abstract}




\section{Introduction}

Because of its simple composition, vast availability in pure form and ease of processing, vitreous silica is often used as a model to study the physics of amorphous solids. In addition to basic science, many studies of amorphous silica are motivated by the fact that it finds widespread use in modern technology, a prominent example being as bulk material in transmissive and diffractive optics for high-power laser applications such as inertial confinement fusion (ICF) $(1,2)$. In these applications, stability under highfluence laser irradiation is a key requirement (3), with optical breakdown occurring when the fluence of the beam is higher than the laser-induced damage threshold (LIDT) of the material (4). Although optical breakdown in glass has been observed for many years, the exact mechanism causing damage remains uncertain. Surface optical breakdown (5) is accompanied by cratering, material ejection, melting and cracking (6-8). Evidence of densification and formation of point defects in the damaged area was also recently documented (9).

Fused silica is used to manufacture transmissive optics for ICF facilities because, of all candidate materials having suitable optical properties, it currently has the highest LIDT at $355 \mathrm{~nm}$ (10). Moreover, there exists a vast body of knowledge on its processing (e.g. melting, grinding, polishing, etc.) and characterization. The development of ever more powerful ICF facilities such as the National Ignition Facility (NIF) in Livermore, 
California and the Laser Méga-Joule near Bordeaux, France provides an incentive for obtaining optical surfaces with even higher LIDT (2).

It has been shown that successive irradiation of existing laser-induced damage sites causes catastrophic damage propagation at fluences lower than the original LIDT (11). Such behavior must be triggered by enhanced coupling between light and the target, for instance due to the formation of micro-cracks at the damage site (12). Propagation of damage in transmissive optics upon repetitive irradiation is currently the most important technological limit in the development of ICF facilities $(2,13)$.

The structure of most $\mathrm{SiO}_{2}$ polymorphs, both crystalline and amorphous, is based on tetrahedral units of silicon coordinated to four oxygen atoms (14). At pressures above 7 gigapascals (GPa) the stable polymorph is stishovite, in which silicon is six-fold coordinated $(15,16)$. Correspondingly, in the 10-20 GPa pressure range, the coordination in glasses increases from 4 to $6(17,18)$. Similarly, the present study shows that under certain conditions laser-irradiated fused silica transforms into a highly defective form of the high-pressure stishovite phase, and this may be responsible for the catastrophic failure caused by repetitive irradiation of the glass.

\section{Experimental procedure}

Polished fused silica windows were damaged on the front surface with 8.4-ns (FWHM) laser pulses from a frequency tripled Nd:YAG laser $(\lambda=355 \mathrm{~nm})$ at a $10 \mathrm{~Hz}$ repetition rate. The laser spot had a Gaussian spatial profile. The $1 / \mathrm{e}^{2}$ spot diameter was 1 
$\mathrm{mm}$. The material surrounding the damage site hole was an aggregate of sub-micron sized redeposited particles (Fig. 1) and could be removed with a solvent leaving the pristine glass surface underneath. The redeposited material was collected from the surface and prepared for transmission electron microscopy (TEM) characterization (Fig. 2) (19). Further characterization was performed by collecting the IR reflection spectrum of the redeposited material and comparing it to the spectra of an unirradiated surface, a pellet of synthetic stishovite and silica powder after compression at $43 \pm 3 \mathrm{GPa}$ in a DAC (Fig. 3) (20).

\section{Results}

\section{1- TEM characterization}

While the majority of the fragments found on the TEM grid were amorphous, some were crystalline. Figure 2 is the scanning TEM (STEM) image of a diffracting fragment, and distinct diffraction rings are clearly seen indicating the presence of small crystallites. Besides silicon and oxygen, other species were present as contaminants: carbon from the carbon film on the grid, fluorine from the HF solution, copper from the TEM grid, and aluminum from the alumina slurry used to finish the optical surface. The relative amount of contamination varied widely within the sample. The spectrum shown in Fig. 2 is therefore not representative of the overall composition of the particle. The $\mathrm{Si} / \mathrm{O}$ ratio could not be measured accurately, nevertheless the fragment was estimated to be nonstoichiometric by comparing its spectrum to a spectrum collected from an untransformed 
glass fragment. The interplanar spacings obtained from the diffraction pattern are compared to those of crystalline stishovite in Table 1. The agreement is good, within experimental accuracy. Furthermore, it must be noted that those spacings cannot be matched satisfactorily to alumina or to any of the known compound salts of aluminum and fluorine, in their anhydrous, hydroxylated or hydrated forms. The diffraction rings are weak and diffuse, indicating that the crystallites are likely to be defective. Moreover, the (100) reflection is normally forbidden for tetragonal stishovite, however a faint ring corresponding to the (100) spacing of stishovite is observed. This is further evidence that the crystals formed are highly defective. Finally, the width of the diffraction rings suggests that a substantial fraction of the crystal population is strained up to a few percent. The crystals seem therefore to contain both chemical (i.e. they are nonstoichiometric) and mechanical defects.

\section{2-IR reflection spectra}

The main features of the IR reflection spectrum of fused silica are indicated in Fig. 3.

The material compressed in the DAC shows a peak at $1383 \mathrm{~cm}^{-1}$ and a broad band at 900 $\mathrm{cm}^{-1}$. The $900 \mathrm{~cm}^{-1}$ band is due to dangling bonds created during compression and comminution (21). Birefringence in the sample due to shear stresses in the DAC causes the $1123 \mathrm{~cm}^{-1}$ glass peak to shift to shorter wavelength, giving rise to the $1383 \mathrm{~cm}^{-1}$ peak in the compressed glass $(22,23)$. Thus IR spectroscopy of the anvil-compressed sample shows no signs of coordination changes upon decompression, in agreement with (17). 
The spectrum of the redeposited material shows the emergence of the stishovite peaks and changes in the glass peaks. The LO component of the $\mathrm{E}_{\mathrm{u}}$ vibration as well as both components of the $A_{2 u}$ vibration of stishovite $(16,24)$ are distinctly visible in the spectrum of the redeposited material (Fig. 3) confirming our interpretation of the TEM results. The position of the new peaks is in good correspondence with those of synthetic stishovite yet the intensity ratios do not match: the intensity of the peaks in the redeposited sample decreases steadily as the wavelength increases. The characteristic $\lambda^{-4}$ decay of peak intensity indicates that the reflectivity of the particles is modulated by Rayleigh scattering (25), as expected for IR-spectra of sub-micron particles. The changes in the glass peaks of the redeposited material occur mostly in the Si-O-Si stretching band: the LO component of this band is enhanced while the TO component nearly disappears. The formation of particles smaller than the IR wavelength is responsible for this effect. Because of its curvature, the entire surface of small particles is not irradiated at normal incidence. Berreman showed that at high incidence angles the LO component of the stretching mode is enhanced (26). A slight shift to lower wavenumbers of the LO component was also noted by Almeida (27). Additional LO enhancement is also due to the electric field distribution in particle/matrix composites (28). IR reflectance data confirm the presence of significant amounts of stishovite crystallites throughout the entire layer of redeposited material. Tetrahedral $\mathrm{SiO}_{2}$ is also detected in the redeposited material. As it is rapidly quenched from high temperature and pressure, the glass is likely 
to contain minute quantities of octahedrally-coordinated silicon (18) that are not detectable by IR spectroscopy. Analysis of the IR spectrum suggests thus that each grain of redeposited material contains stishovite micro-cystallites in a matrix of tetrahedral silica glass. The porosity of the diffracting particle after HF etching (Fig. 1) confirms this morphology.

\section{Discussion}

Laser-induced surface breakdown is accompanied by the formation of a plasma in front of the target surface. Since it forms during the pulse, the plasma absorbs the laser energy and shields the target surface $(6,8)$. As a result, the pressure at the target is much lower than in the plasma. Energy is supplied to the plasma during the laser pulse (8.4 ns) when only limited expansion takes place. Therefore, initially the plasma is a hot, partially ionized (29) fluid of near-solid density. It is thus likely that the stishovite crystals form in the plasma during or shortly after the laser pulse, before the expansion causes a rapid decrease in density, temperature and pressure of the fluid. The pressure in the plasma must be greater than $7 \mathrm{GPa}$ for stishovite to be stable (30). In similar pressure conditions (e.g. shock loading) other crystalline $\mathrm{SiO}_{2}$ polymorphs, quartz and cristobalite, were observed to form a tetrahedral diaplectic glass (31-33) and to transform into stishovite only when copper particles were added to the initial mixture in order to increase the thermal quenching rate (34). Here we show that stishovite crystals form under laser irradiation in time scales ( few tens of nanoseconds) comparable to those characteristic 
of typical shock-loading experiments (nanoseconds to microseconds). The higher temperature and the presence of ionized species in the plasma might favor the more ionic $\mathrm{SiO}_{2}$ polymorph (stishovite) through Coulomb interactions. The formation of crystalline phase with octahedrally coordinated silicon is in agreement with network topology: the low degree of structural freedom of such a structure hinders the formation of an amorphous phase (35). Moreover, crystallization of silica into stishovite under laser irradiation may be kinetically facilitated by the high surface energy associated with the formation of small particles in the plasma. Contrary to shock loading, the transformation is mediated by the formation of a high-energy fluid phase and does not necessarily have to proceed through an all-solid-state path, favoring the formation of the crystalline phase over the amorphous. Finally, the high quenching rate allowed by laser irradiation prevents the stishovite crystals to back-transform into silica. This phase transformation has profound effects on the properties of the material as the coordination change of the central silicon atom is accompanied with an alteration of the nature of the silicon-oxygen bond from almost purely covalent into partially ionic (17).

\section{Conclusion}

Stishovite is customarily formed by shock-loading or by quasi-statically compressing low-pressure $\mathrm{SiO}_{2}$ phases in an effort to simulate the conditions under which stishovite is formed in nature (e.g. planetary interiors or meteorite impacts). In the present work, we show for the first time that laser illumination conditions that are representative of those 
used in ICF facilities (36) can induce this phase transformation in optical grade synthetic silica.

Laser-driven phase transformation of fused silica into stishovite has important implications in terms of laser damage propagation in optics used in high-power laser facilities. Stishovite has a much higher refractive index $(\mathrm{n} \approx 1.8)(15)$ than silica $(\mathrm{n} \approx 1.46)$ and can introduce significant micro-lensing effects. During repetitive irradiation, microlensing at a damaged site can create localized field enhancements that may exceed the LIDT of the surrounding material, thus providing a mechanism for optical breakdown propagation. More importantly, stishovite has the smallest bandgap $(5.5 \mathrm{eV})$ of all $\mathrm{SiO}_{2}$ polymorphs (37). Its bandgap is lower than the energy of two 355-nm photons. The change from a three-photon to a two-photon absorption mechanism substantially lowers the threshold for multi-photon ionization, which is a possible energy absorption mechanism for damage propagation. Moreover, the observed presence of defects in the stishovite crystals is likely to cause intra-gap absorbing states that could further lower the LIDT of damaged fused silica at all wavelengths. Finally, the optical properties of the untransformed glass may also be altered by the presence of small amounts of octahedrally-coordinated silicon quenched in the amorphous structure. If damage propagation is indeed due to the formation of stishovite, a successful growth mitigation strategy may include local annealing (e.g. with $\mathrm{CO}_{2}$ laser radiation) of a damage spot in order to back-transform the stishovite into tetrahedral $\mathrm{SiO}_{2}$ glass. 


\section{References}

1. E. M. Campbell, Fusion Technology 26, 755 (1994).

2. J. Glanz, Science 277, 304 (1997).

3. Laser-Induced Damage in Optical Materials (SPIE/NBS-SP, 1969-2001).

4. N. Bloembergen, IEEE Journal of Quantum Electronics QE-10, 375-86 (1974).

5. N. L. Boling, M. D. Crisp, G. Dubé, Applied Optics 12, 650-60 (1973).

6. M. Von Allmen, A. Blatter, Laser-Beam Interactions with Materials. (Springer, New York, 1995).

7. N. L. Boling, G. Dubé, Applied Physics Letters 21, 487-489 (1972).

8. A. Salleo, F. Y. Génin, M. D. Feit, A. M. Rubenchik, T. Sands, S. S. Mao, R. E. Russo, Applied Physics Letters 78, 2840 (2001).

9. M. A. Stevens-Kalceff, A. Stetsmans, J. Wong, Applied Physics Letters 80, 758-760 (2002).

10. L. L. Chase, in Laser Ablation J. C. Miller, Ed. (Springer-Verlag, Berlin Heidelberg, 1994), vol. 28, pp. 53-82.

11. A. Salleo, T. Sands, F. Y. Génin, Applied Physics A A71, 601-8 (2000).

12. F. Y. Génin, A. Salleo, T. V. Pistor, L. L. Chase, Journal of the Optical Society of America A 18, 2607 (2001).

13. C. Seife, D. Malakoff, Science 289, 1126 (2000). 
14. W. D. Kingery, H. K. Bowen, D. R. Uhlmann, Introduction to Ceramics. (Wiley, New York, 1976).

15. S. M. Stishov, S. V. Popova, Geokhimya 10, 837-839 (1961).

16. A. M. Hofmeister, J. Xu, S. Akimoto, American Mineralogist 75, 951-955 (1990).

17. Q. Williams, R. Jeanloz, Science 239, 902-5 (1988).

18. X. Xue, J. F. Stebbins, M. Kanzaki, R. G. Tronnes, Science 245, 962-4 (1989).

19. The collected material was soaked in a diluted HF solution (5\%) overnight. During the $\mathrm{HF}$ etching, tetrahedral $\mathrm{SiO}_{2}$ dissolves in the acid according to Kleeman and Ahrens, J. Geophys. Res. 78(26), 5954 (1973). The remaining material was rinsed and suspended in ethanol. A drop of the ethanol suspension was deposited on a copper grid coated with holey carbon suitable for TEM. TEM characterization was performed on a Philips CM200 FEG-(S)TEM equipped with a field emission gun and operating at $200 \mathrm{kV}$. Energy dispersive X-ray spectra were acquired in scanning mode using a focused probe. Probe positioning was facilitated using annular dark-field images acquired in scanning TEM mode. The analyzed fragment damaged under electron beam irradiation therefore yielding only a qualitative analysis of the composition.

20. The IR spectra were taken with a Nic-Plan IR microscope equipped with a liquid nitrogen cooled MCT detector connected to a Nicolet 760 FTIR spectrometer. The radiation source was a thermal black body. The frequency range was $550 \mathrm{~cm}^{-1}$ to 10,000 
$\mathrm{cm}^{-1}$. The scan resolution was $0.5 \mathrm{~cm}^{-1}$. The spot diameter at the sample was approximately $100 \mu \mathrm{m}$.

A synthetic stishovite pellet was prepared by hot isostatic compression of vitreous silica in a graphite furnace. Transformation of glass into stishovite was verified by X-ray diffraction.

Synthetic silica from the same batch as the irradiated sample was ground to $<1$ micron grains and compressed in a DAC. 2-3 ruby chips were added to the sample for pressure measurement according to H. K. Mao et al., Journal of Applied Physics 49, 3276 (1978).

21. P. Dean, Reviews of Modern Physics 44, 127-68 (1972).

22. A shift exceeding $200 \mathrm{~cm}^{-1}$ is not likely to be due to structural transformation in the glass as hydrostatic pressure-induced shifts in the $1123 \mathrm{~cm}^{-1}$ peak are usually limited to a few tens of wavenumbers: see for example B. Velde and R. Couty, Chemical Geology 62, 35 (1987).

23. V. Nazabal, E. Fargin, G. Le Flem, V. Briois, C. C. D. Moulin, T. Buffeteau, B. Desbat, Journal of Applied Physics 88, 6245-6251 (2000).

24. Q. Williams, R. J. Hemley, M. B. Kruger, R. Jeanloz, Journal of Geophysical Research-Solid Earth 98, 22157-22170 (1993).

25. H. C. Van De Hulst, Light Scattering by Small Particles. (Dover Publications, New York, 1981).

26. D. W. Berreman, Physical Review 130, 2193 (1963). 
27. R. M. Almeida, Physical Review B 45, 161-170 (1992).

28. S. M. Hu, Journal of Applied Physics 51, 5945-8 (1980).

29. L. Escobar-Alarcon, M. Villagran, E. Haro-Poniatowski, J. C. Alonso, M. Fernandez-

Guasti, E. Camps, Applied Physics A A69, S583-S586 (1999).

30. F. R. Boyd, J. L. England, Journal of Geophysical Research 65, 752 (1960).

31. A. J. Gratz, L. D. Deloach, T. M. Clough, W. J. Nellis, Science 259, 663-666 (1993).

32. K. J. Kingma, C. Meade, R. J. Hemley, H. K. Mao, D. R. Veblen, Science 259, 666669 (1993).

33. A. J. Gratz, W. J. Nellis, J. M. Christie, W. Brocius, J. Swegle, P. Cordier, Physics and Chemistry of Minerals 19, 267-288 (1992).

34. J. D. Kleeman, T. J. Ahrens, Journal of Geophysical Research 78, 5954-60 (1973).

35. L. W. Hobbs, A. N. Sreeram, C. E. Jesurum, B. A. Berger, Nuclear Instruments \& Methods in Physics Research B 116, 18-25 (1996).

36. E. Breval, M. Alam, S. Kwan, M. Grutzeck, Journal of Materials Science Letters 10, 765-767 (1991).

37. J. R. Alvarez, P. Rez, Solid State Communications 108, 37-42 (1998).

38. J. C. Decius, R. M. Hexter, Molecular Vibrations in Crystals. (McGraw-Hill, New York, 1977).

39. J. Wong, C. A. Angell, Glass : Structure by Spectroscopy. (M. Dekker, New York, 1976). 
Acknowledgements: synthetic stishovite was provided by Baosheng $\mathrm{Li}$ at State University of New York, Stony Brook. Work performed at the National Center for Electron Microscopy (TEM characterization) and at the Advanced Light Source (IR spectroscopy) at Lawrence Berkeley National Laboratory was supported by the Director, Office of Science, Office of Basic Energy Sciences, Material Sciences Division of the U.S. Dept. of Energy under contract No. DE-AC03-76SF00098. Work by two of the authors (A. S. and F. Y. G.) was performed under the auspices of the U.S. Department of Energy at Lawrence Livermore National Laboratory under contract No. W-7405-Eng-48. 


\begin{tabular}{|c|c|c|}
\hline Measured spacing & \multicolumn{2}{|c|}{${\text { Stishovite } \mathbf{d}_{\text {hkl }}}^{\text {Crystal plane }}$} \\
\hline $4.25 \pm 0.25$ & 4.17 & $(100)$ \\
$2.9 \pm 0.1$ & 2.96 & $(110)$ \\
$2.68 \pm 0.08$ & 2.66 & $(001)$ \\
$2.25 \pm 0.08$ & 2.25 & $(101)$ \\
$2.08 \pm 0.05$ & 2.09 & $(200)$ \\
$1.89 \pm 0.06$ & 1.87 & $(210)$ \\
$1.60 \pm 0.05$ & 1.53 & $(211)$ \\
\hline
\end{tabular}

Table 1: Interplanar spacings measured from TEM diffraction patterns (Fig. 2) and spacings of stishovite for various crystallographic planes. 


\section{List of Figures}

Figure 1: Photograph and SEM micrograph (inset) of the material redeposited around the drilled hole. Damage was initiated on the front surface with a $45 \mathrm{~J} / \mathrm{cm}^{2}$ laser burn. The laser-induced surface breakdown generated a plasma and an audible explosion. Repetitive irradiation at lower fluence caused laser drilling from the surface into the bulk of the window. Material was found redeposited at the surface within the footprint of the plasma in a 3-mm radius around the entrance hole of the drilled channel.

Figure 2: STEM micrograph, ring diffraction pattern and EDS spectrum of a crystalline particle of the redeposited material. Signal from the TEM grid is indicated in dashed line.

Figure 3: IR reflectance spectra of the pristine surface, statically compressed silica, and redeposited material. The spectrum of synthetic stishovite is included for comparison. The $\lambda^{-4}$ decay due to Rayleigh scattering is indicated in the figure. The main features of the IR reflection spectrum of fused silica are a peak centered around $790 \mathrm{~cm}^{-1}$ attributed to Si-O-Si rocking motion and a strong peak at $1123 \mathrm{~cm}^{-1}$ with a shoulder at $1236 \mathrm{~cm}^{-1}$ due to the splitting of the longitudinal optical and transverse optical (LO-TO) components of the asymmetric stretching of tetrahedral $\mathrm{Si}-\mathrm{O}$ units $(38,39)$. The spectrum of the redeposited material shows changes in the glass peaks and the emergence of new peaks. The intensity of the $1236 \mathrm{~cm}^{-1}$ shoulder increases until it becomes a separate peak. This peak also narrows and shifts to higher wavenumbers reaching $1258 \mathrm{~cm}^{-1}$. The intensity of the main $1123 \mathrm{~cm}^{-1}$ peak decreases until almost vanishing. A new peak 
emerges at $1035 \mathrm{~cm}^{-1}$ and progressively shifts to $1020 \mathrm{~cm}^{-1}$. Other weaker new peaks can be seen at $875 \mathrm{~cm}^{-1}$ and $665 \mathrm{~cm}^{-1}$. 


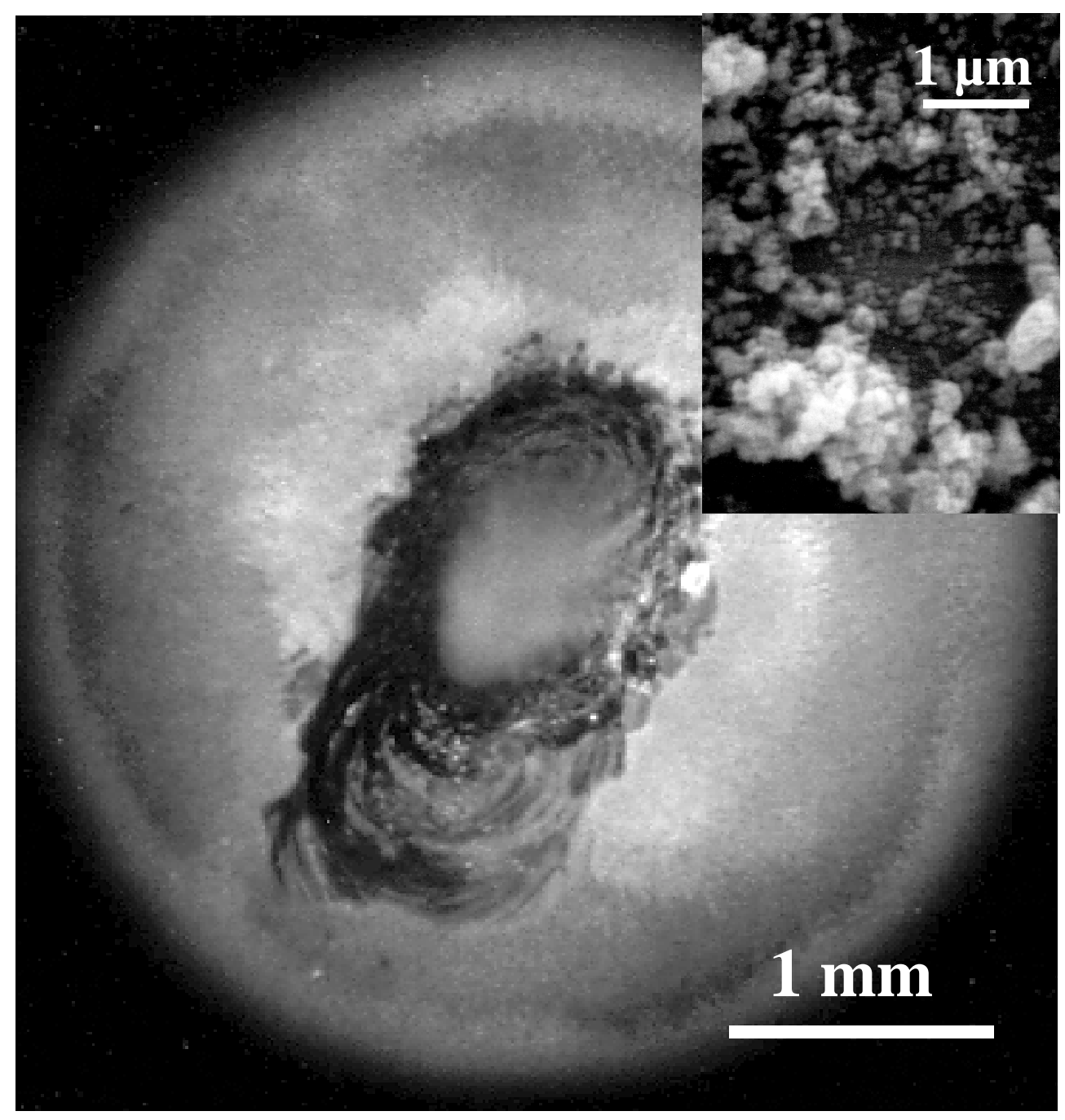

Figure 1 


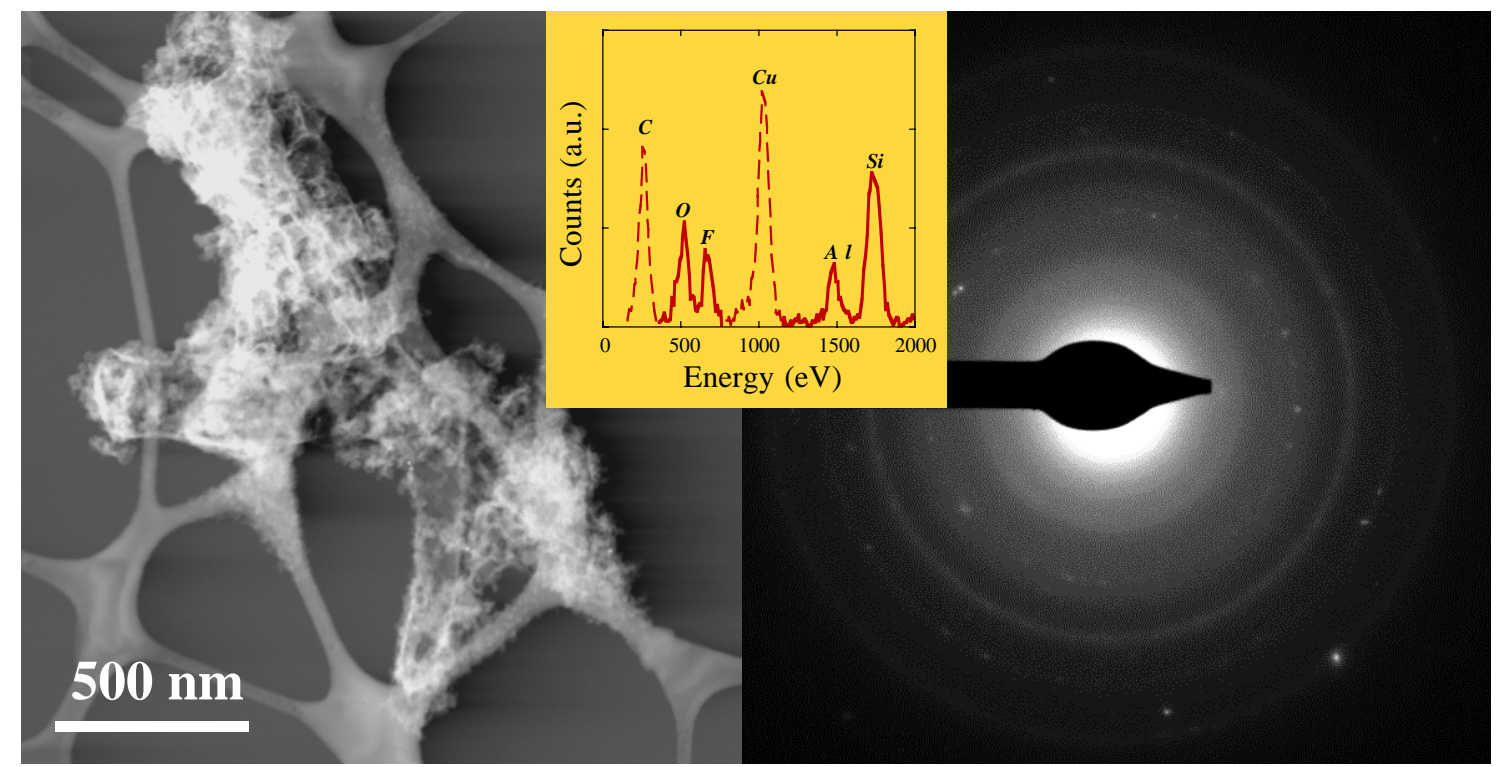

Figure 2 


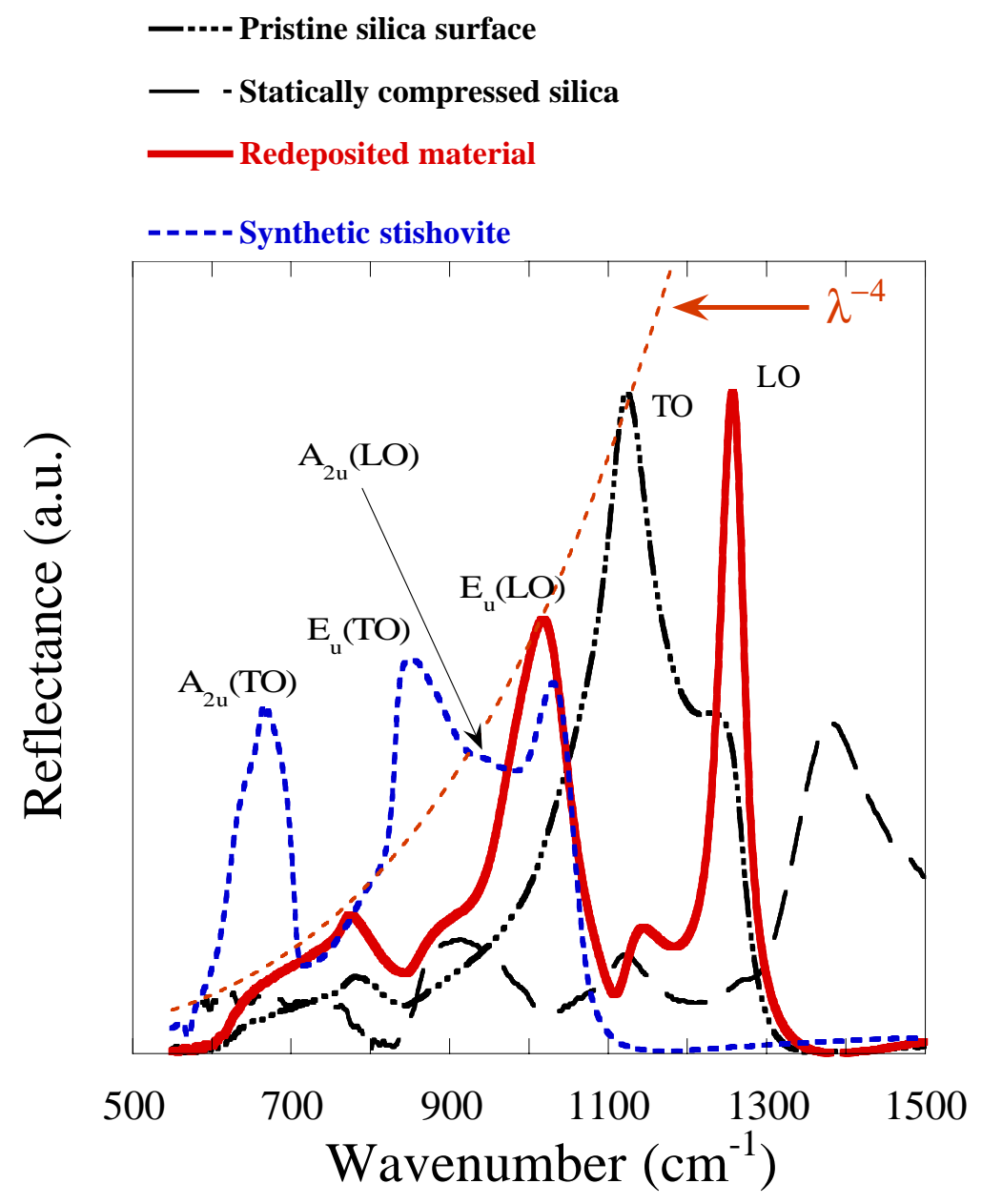

Figure 3 